\title{
GLUCAGON LIKE PEPETIDE-1 AGONIST IN A PATIENT WITH PRADER-WILLI SYNDROME: A SRI LANKAN EXPERIENCE
}

\section{Maulee Hiromi Arambewela, Noel P. Somasundaram}

National Hospital Sri Lanka.

\section{ABSTRACT}

\section{Background}

Prader-Willi Syndrome (PWS) is a genetic disease characterized by morbid obesity and hyperphagia. Although the exact pathophysiological basis of obesity and hyperphagia remains unclear it is thought to be mainly due to hypothalamic dysfunction. Currently the options for medical therapy are limited and most are undergoing clinical trials. Lately glucagon like peptide agonist -1(GLP-1 agonist) has been tried out in this patient population due to its role in causing weight reduction, appetite suppression and glycemic control. We discuss our experience in using GLP-1 agonist on an adolescent with PWS and the challenges faced in managing these patients in a developing nation.

\section{Case Presentation}

A 16 year old Sri Lankan girl with PWS, presented with short stature, primary amenorrhea, morbid obesity (BMI $=60)$ and hyperphagia. She had defaulted clinic follow up during child hood. Investigations revealed hypogonadotrophic hypogonadism and growth hormone deficiency. Cortisol, prolactin and thyroid hormonal levels were normal as was the imaging of the pituitary gland. Glycemic assessment revealed pre diabetes with $\mathrm{HbA} 1 \mathrm{c}$ level of $6.2 \%$ and fasting blood glucose $120 \mathrm{mg} / \mathrm{dl}$. She was commenced on liraglutide monotherapy which resulted in remarkable control of hyperphagia. She lost 3 kilograms during a period of three months and HbA1c dropped from $6.2 \%$ to $5.0 \%$. However due to the high cost of the drug, her caregiver was compelled to discontinue it after 3 months of treatment. She was commenced on replacement of growth hormone, estradiol and metformin. However, hyperphagia recurred and she regained weight. Attempts to refer the patient for behavioral therapy were declined by the patient and her caregiver.

\section{Conclusion}

GLP-1 agonists seem to be a promising drug for hyperphagia and morbid obesity in patients with PWS and warrants further study to demonstrate its efficacy and safety in the long term. Inaccessibility to modern and expensive drugs, social stigma in seeking psychiatric and behavioral therapies, lack of support groups and organized institutional care for such patients are some of the obstacles in managing a rare and challenging disease like PWS in developing countries where the ever rising communicable and non communicable diseases drain the bulk of the health budget.

Running title: Delayed adrenache in thalassaemia

Keywords: Thalassaemia, iorn overload, delayed adrenache, delayed puburache.

\section{INTRODUCTION}

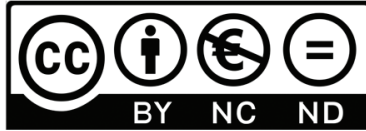

Prader Willi Syndrome (PWS) is a common genetic cause of childhood obesity with a prevalence rate of $1: 10,000-1: 25,000^{1}$. It is the first human disorder to 
be recognized as related to genomic imprinting due to the failure of expression of paternal genes presented in the region of chromosome $15 q 11.2$-q132. The disease is characterized by hypotonia at birth, feeding difficulties during infancy, developmental delay, intellectual disability, childhood onset of obesity with hyperphagia, endocrine dysfunction and neuropsychiatric issues. The diagnosis is suspected based on clinical features and confirmed by genetic studies(3). The endocrinological, cognitive and behavioural alterations occurring in PWS is secondary to hypothalamic dysfunction.

As the underlying pathophysiological basis for PWS phenotype is unclear, current therapies have shown limited efficacy and are directed at symptomatic control only. Growth hormone (GH) replacement is a standard of care for children as well as some adults as deficiency of this hormone is present in almost all children and most adults with PWS(4). Replacement therapy results in improvement of height, body composition, bone mineral density, exercise endurance and causes a favorable impact on development and behavior(5). However it does not tackle hyperphagia and morbid obesity which is one of the most challenging issues in managing these patients. Typical overweight or obesity results from a small error in average daily energy balance. In contrast children with PWS have been known to ingest 6 times their daily energy requirement(7). Caretakers of adults with PWS have documented 30 pounds of weight gain over a matter of days when patients had free access to food(4).

The exact molecular mechanisms as to the occurrence of obesity and hyperphagia in PWS remain incompletely understood. The satiety hormone, "Leptin" is found in normal levels in these patients but their hypothalamus seems to be unresponsive to its effect. Studies have found out that "Ghrelin", the hunger hormone is high in these patients even after a meal. However the hypothalamus seems to be insensitive to this hormone as well(8). Other studies have shown defects in hypothalamic neuropeptides such as oxytocin, hypocretin/orexin as possible causes for hyperphagia in these patients(9).

At present there is an urgent need for pharmacological options as constant supervision is demanding and distressing for both the patient and the caregiver and surgical options such as bariatric surgery are contraindicated in this population.

Glucagon like peptide agonist -1(GLP-1 agonist) (liraglutide and exenatide) were originally developed for the treatment of patients with type 2 diabetes mellitus. Secreted by the L cells in the small intestine, in response to nutrient ingestion, GLP-1 agonists, increase Insulin secretion, and suppresses glucagon in a glucose dependent manner. It decreases food intake by delaying gastric emptying and increasing satiety. GLP-1 agonists stimulate satiety by binding to its receptor in the hypothalamus and reduce calorie ingestion by delayed gastric emptying(10). This drug has shown to reduce appetite and induce weight loss in both diabetic and non-diabetic populations(11).

We present a case highlighting our experience with Glucagon-like peptide-1(GLP 1) receptor agonists in an adolescent with PWS and the challenges encountered in managing these patients in a developing nation.

\section{CASE PRESENTATION}

A 16 year old Sri Lankan female presented with short stature, obesity and primary amenorrhea. She had been diagnosed as Prader Willi Syndrome (PWS) during early child hood but had defaulted medical follow up. She has had feeding problems due to poor sucking during infancy and has had to be breastfed. All her developmental milestones had been delayed and although attending a normal school, her performance was far below average. She had suffered from hyperphagia and behavioral issues regarding controlling of feeding since early child hood.

She was morbidly obese with a height of $130 \mathrm{~cm}\left(<3^{\text {rd }}\right.$ centile), weight of $102 \mathrm{~kg}$ ( $>97^{\text {th }}$ centile) and Basal Metabolic Index (BMI) of 60. She had the characteristic facial appearance of PWS, consisting of almond eyes with strabismus, thin upper lip with down turned mouth and small hands and feet. Acanthosis nigricans was evident. She had inadequate development of secondary sexual characteristics with Tanner 2 staging for both breast and pubic hair.

Investigations revealed pre diabetes with $\mathrm{HbA} 1 \mathrm{c}$ of $6.2 \%$ and fasting blood glucose of $120 \mathrm{mg} / \mathrm{dl}$. 
Hormonal evaluation revealed hypogonadotrophic hypogonadism with low serum estradiol of $18 \mathrm{pg} / \mathrm{ml}$ (21-312) and low levels of Luteinizing hormone 0.1 U/L (1.9-12.5) and Follicular Stimulating Hormone 0.69 U/L (2.5-10.2). Serum Insulin like Growth Factor (IGF -1) levels were low at $130 \mathrm{ng} / \mathrm{ml}$ (age matched reference range 226-903) and subsequent Insulin tolerance Test confirmed growth hormone deficiency with sufficient levels of cortisol. She was clinically and biochemically euthyroid with serum free Thyroxin levels of $1.29 \mathrm{ng} / \mathrm{dl}$ ( 0.8-1.6) and Thyroid Stimulating Hormone levels of $1.09 \mathrm{mU} / \mathrm{l}$ (0.35-5.5). Prolactin levels were normal at 196mU/L (60-620). Imaging of the pituitary was normal.

Figure 1 - Patient with PWS

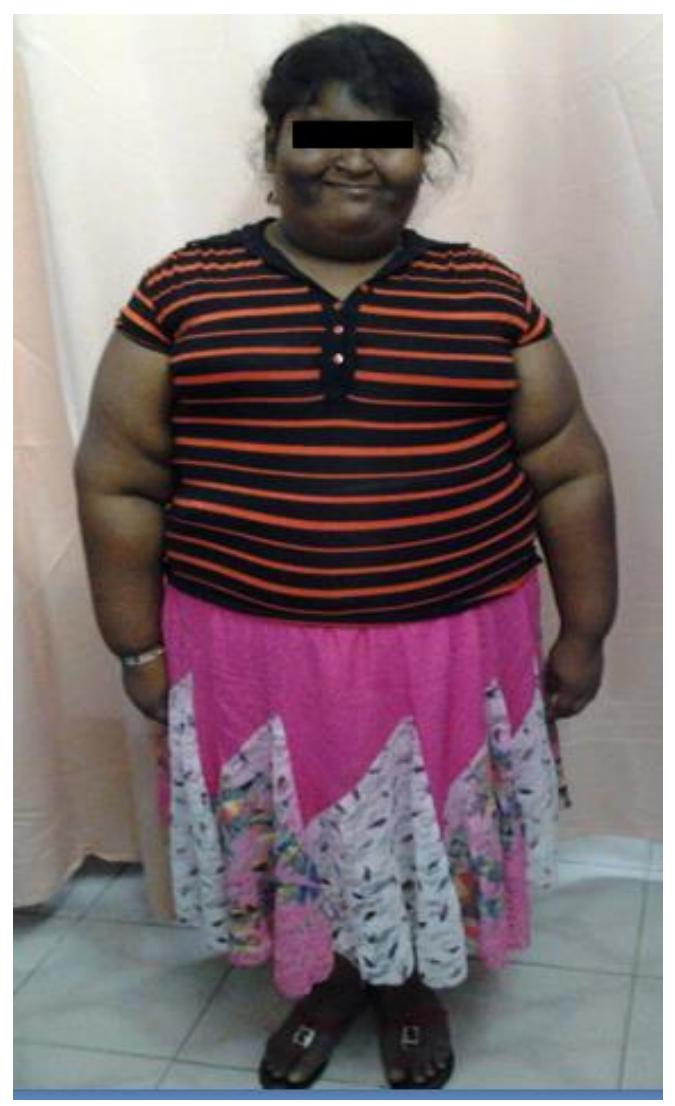

As the primary concern of her caregiver was obesity and overeating which was difficult to control, it was decided to give her a trial of liraglutide. She was commenced on sub cutaneous liraglutide $0.6 \mathrm{mg}$ daily for two weeks which was subsequently increased to
$1.2 \mathrm{mg}$ daily. She was referred for counseling and behavioural therapy for overeating was but had defaulted their follow up. During the three months following commencement of liraglutide, the patient experienced a marked change in behavior. Her appetite diminished and she lost 3 kilograms. Her HbA1c improved from $6 \%$ to $5 \%$. However her caregiver was finding it increasingly difficult to afford liraglutide which is not supplied free from the statesector hospitals. Thus unfortunately the drug was discontinued. She was commenced on replacement of growth hormone, and estradiol as well as metformin. However hyperphagia recurred and she regained her lost weight over the next 4 months. Counseling and behavioral therapy was emphasized again but declined by the patient and her caregiver.

\section{DISCUSSION}

Our patient had a good response to GLP-1 agonists by demonstrating a $3 \mathrm{~kg}$ weight loss over a three month period and showed a favorable reduction in HbA1c. However the most remarkable effect was the reduction in appetite leading to better control of her eating habits.

Lately GLP-1 agonists have been considered as a new therapeutic option in tackling hyperphagia and obesity among PWS patients. This is due to its potential effects on ghrelin suppression, central appetite suppression and increase in the energy expenditure and stimulation of insulin secretion(12).

Several studies have looked at its therapeutic utility in patients with PWS. A small placebo controlled cross over study done on the effects of a single dose of exenatide on appetite, gut hormones and glucose homeostasis in adult patients with PWS and obese controls, demonstrated increase in satiety independent of measured appetite hormones, lower glucose levels and insulinotropic effect in both groups(12). Ghrelin levels and energy expenditure were not affected and gastrointestinal side effects were common among the obese patients but absent among patients with PWS. Similarly the drug was well tolerated in our patient as well. A Case reported from Japan of a patient with PWS on liraglutide therapy for one year duration, demonstrated suppression of appetite throughout the treatment duration and favorable reductions in body weight, visceral fat, HbA1c and Ghrelin levels(13). Fintinin et al reported 
improvement or stabilization of altered glucose metabolism and clinical parameters more evident during the first year of treatment in 6 patients with PWS who were treated with GLP-1 agonists for 2 years(14). All these studies demonstrated that liraglutide was well tolerated. This is postulated to be due to the high threshold for pain and nausea among these patients. However more studies on the dosing and duration of treatment are warranted.

Concerns of liraglutide include its potential to delay gastric emptying, with a theoretical risk of gastric rupture in a patient population vulnerable to the risk of hyperphagia induced injuries and even death(15). Unpublished pilot studies have shown that GLP-1 agonists further exacerbates the slow gut movements in patients with PWS. This warrants careful supervision of patients on GLP-1 agonists. At present, GLP-1 agonists are undergoing phase 2 clinical trials and are not yet routinely prescribed(4). Miller et al looked in to various types of medication inclusive of GLP-1 agonists, oxytocin analogues and ghrelin analogues which are currently undergoing clinical trials. However as of today there is no drug which has been proven to be effective for regulation of hyperphagia in these patients.

Our patient was able to take the drug for 3 months, during which time she developed marked reduction of appetite enabling her to reduce $3 \mathrm{~kg}$ of the body weight and favourable reduction in HbA1c. However liraglutide is currently not provided by the state sector, thus necessitating patients to purchase it. Unfortunately, due to its high cost liraglutide had to be discontinued, following which recurrence of hyperphagia and weight gain occurred. Attempts to refer her again for behavioral therapy were also not successful.

PWS is a challenging disease which needs a multidisciplinary approach to successful management. Inaccessibility to modern and expensive drugs, social stigma against seeking psychiatric and behavioral therapies, lack of support groups and organized institutional care for such patients are some of the obstacles faced by developing countries where the ever rising communicable and non-communicable diseases drain the bulk of the health budget. Thus rare and complex diseases such as PWS tend to be overlooked. However as PWS is increasingly being diagnosed in infancy, early, efficient and cost effective intervention for tackling issues such as hyperphagia and obesity would invariably improve the quality of life of these patients.

\section{CONCLUSION}

PWS is a complex disease with hyperphagia and obesity causing much morbidity and indirect mortality. At present there are no efficient cost effective medical options which are routinely used to tackle this problem. Our patient with PWS who was treated with liraglutide for 3 months demonstrated remarkable improvement in hyperphagia, weight loss and glycemic control. However due to the high cost of liraglutide, therapy had to be discontinued resulting in recurrence of hyperphagia and weight gain. This case highlights the potential benefits of GLP-1 agonists in these patients and obstacles faced by developing nations in tackling such diseases.

\section{ABBREVIATIONS}

PWS - Prader Willi Syndrome

GH- Growth hormone

(GLP 1 agonist ) - Glucagon-like peptide-1 agonist

BMI - Basal Metabolic Index

\section{REFERENCES}

1. Burman $P$, Ritzen EM, Lindgren AC. 2001Endocrine dysfunction in Prader-Willi syndrome: a review with special reference to $\mathrm{GH}$. Endocr Rev 2001; 22:787-799

2. Butler MG. Prader-Willi syndrome: obesity due to genomic imprinting. Curr Genom. 2011;12(3):204-215 doi:10.2174/138920211795677877.

3. Gunay-Aygun M, Schwartz S, Heeger S, O’Riordan MA, Cassidy SB. The changing purpose of PraderWilli syndrome clinical diagnostic criteria and proposed revised criteria. Pediatrics. 2001;108:E92

4. Miller JL, Strong TV, Heinemann J. Medication trails for food related behavior in Prader-Willi Syndrome. Diseases 2015; 3:78-85 doi:10.3390/diseases3020078

5. Deal C.L, Tony M, Höybye C, Allen DB, Tauber M, Christiansen J.S. Growth Hormone in Prader-Willi Syndrome Clinical Care Guidelines Workshop Participants. Growth Hormone Research Society 
workshop summary: Consensus guidelines for recombinant human growth hormone therapy in Prader-Willi syndrome. J. Clin. Endocrinol. Metab. 2013;98:E1072-E1087, doi:10.1210/jc.20123888.

6. Gourash LM, Forster JL. Regulation of weight in Prader-Willi syndrome: Theoretical and Practical consideration. Pittsburgh partnership 2009

7. Zipf WB, Bentson GG. Characteristics of abnormal food intake. Am J Clin Nutr 1987 46:277-281)

8. Feigerlová E, Diene G, Conte-Auriol F, Molinas C, Gennero I, Salles JP, Arnaud C, Tauber M. Hyperghrelinemia precedes obesity in PraderWilli syndrome. J Clin Endocrinol Metab. 2008. doi:10.1210/jc.2007-2138

9. Swaab, D.F.; Purba, J.S.; Hofman, M.A. Alterations in the hypothalamic paraventricular nucleus and its oxytocin neurons (putative satiety cells) in Prader-Willi syndrome: A study of five cases. $J$. Clin. Endocrinol. Metab. 1995, 80, 573-579

10. S. E. Kanoski, L. E. Rupprecht, S. M. Fortin, B. C. de Jonghe, and M. R. Hayes, "The role of nausea in food intake and body weight suppression by peripheral GLP-1 receptor agonists, exendin-4 and liraglutide," Neuropharmacology. 2012;62(5);1916-1927, 2012.

11. Rosenstock J, Klaff LJ, Schwartz S,Northup J, Holcombe JH,Wihem K, Trautmann M. Effects of exenatide and life style modification on body weight and glucose tolerance in obese subjects with and without pre diabetes. Diabetes Care 2010;33:1173-1175

12. Sze L,Purtell L, Jenkins A, et al. Effect of single dose in Exenatide on appetite, gut hormones and glucose homeostasis in adults with Prader-Willi syndrome. $J$ CLin Endocrinol Metab. 2011,96(8)E1314-1319.

13. Senda, $M$, Ogawa $S$, Nako, $K$, Okamura $M$, Sakamoto T, Ito S. The glucagon-like peptide-1 analog liraglutide suppresses ghrelin and controls diabetes in a patient with Prader-Willi syndrome. Endocr. J. 2012;59:889-894

14. Fintini D,Grugni G, Brufanic C et al. Use of GLP-1 Receptor Agonist in Prader-Willi Syndrome. Report of six cases. Diabetes Care 2014;37:e7677.

15. Stevenson DA, Heinemann J, Angulo M, etal. Gastric rupture and necrosis in Prader-Willi syndrome. $J$ Pediatr Gastroenterol Nutr 2007;45:272-274 